\title{
DISCONTINUANCE OF ADHD TREATMENT IN ADOLESCENTS
}

Prevalence of ADHD drug discontinuance in adolescents and young adults was studied in the UK by using the General Practice Database for patients aged 15-21 years from 1999 to 2006. Prevalence of prescribing methylphenidate, dexamfetamine and atomoxetine across all ages increased 6-fold over the study period, but overall, prevalence decreased with age. In 2006, prevalence of prescribing in males dropped 95\% from 12 per 1000 in 15-yearolds to 0.64 per 1000 in 21-year-olds. A longitudinal analysis of 44 patients aged 15 years in 1999 found no patient received treatment after age 21 years. The fall in prescribing by general practitioners in UK is greater than the reported age-related decrease in ADHD symptoms. (McCarthy S, Asherson P, Coghill D, et al. Attention-deficit hyperactivity disorder: treatment discontinuation in adolescents and young adults. Brit J Psychiatry March 2009;194:273-277). (Respond: Dr Ian CK Wong, Institute of Child Health, University College London, 29/39 BrunswickSquare, London WC1N 1AX, UK. E-mail: ian.wong@pharmacy.ac.uk).

COMMENT. The recent rapid increase in prescriptions for patients with ADHD in the UK is of interest, since practitioners in the UK have generally denied the existence of the syndrome and disapproved of its "medicalization." At a meeting of pediatric neurologists in London in the 1970 s, I was gently chided by my British colleagues for my interest in treatment of the behavior. Rutter $\mathrm{M}$ et al, in a study of 10- and 11-year-old children in the Isle of Wight, UK, reported a prevalence of less than 1 in 1000. (Education, Health and Behavior. London, Longman, 1970). In contrast, prevalence rate among school-age children in the US is estimated at 5\%, and even higher when using the latest DSM diagnostic criteria.(Wolraich ML et al. J Am Acad Child Adolesc Psychiatry 1996;35:319-324). The decreased prevalence of prescribing noted in adolescents and young adults in the UK study is also noted among adults in a Hungarian study (Simon V et al. Brit J Psychiatry 2009; 194:204-211). The pooled prevalence of adult ADHD was 2.5\%, and this decreased with increasing age. The reasons for the decline in prevalence of treated ADHD in young adults are unclear. Possible explanations include inadequate diagnostic criteria, and a paucity of neurologists and psychiatrists who address the problems of that transition period from late adolescence to adulthood. Pediatric neurologists, trained both in adult and pediatric medicine, are eminently qualified to care for this age group, but the geographic separation of the specialties may present an obstacle.

\section{CEREBELLAR ATAXIAS}

\section{PONTOCEREBELLAR HYPOPLASIA TYPE 1}

Slowly progressive cerebellar ataxia in a 12-year-old Indian boy with early onset anterior horn cell spinal muscular atrophy is reported from the Department of Pediatric Neurology, Government Medical College, Kerala, India. Born of nonconsanguineous parents, he was hypotonic at birth, milestones were delayed, and he had frequent respiratory infections and fluid aspirations. At 12 years, he walked with a wide based ataxic gait, his muscles were wasted and hypotonic, and reflexes were absent. Bilateral gaze nystagmus, past 
pointing, and dysdiadochokinesia were noted. EMG showed fibrillations, fasciculations, and polyphasic potentials, and muscle biopsy showed evidence of denervation, with variation in fiber size and group atrophy compatible with spinal muscular atrophy type 3. MRI showed marked atrophy of the cerebellar vermis and hemispheres, and prominent cisterna magna and prepontine cisterns. Molecular genetic testing for SMN1 on chromosome 5q showed no deletions at exon 7 and 8. Mitochondrial disease was excluded. Serum transferrin test for carbohydrate-deficient glycoprotein syndrome was not available. This case represents a mild variant of pontocerebellar hypoplasia type 1. (Kalpana D, Parvathy L, Ahamed SM, Iype M, Kunju MPA. A mild variant of pontocerebellar hypoplasia type 1 in a 12-year-old Indian boy. Pediatr Neurol April 2009;40:302-305). (Respond: Dr Kalpana. E-mail: vijaykal@hotmail.com).

COMMENT. In the diagnostic work-up of a child with slowly progressive cerebellar ataxia, a large spectrum of neurodegenerative and metabolic disorders needs to be considered. Hereditary and sporadic ataxias are the two major categories. Hereditary cases are autosomal dominant, autosomal recessive, X-linked, or mitochondrial. Sporadic cases are symptomatic (structural lesions, developmental, neoplastic, toxic, drug-induced, endocrine [hypothyroidism], malabsorption [celiac disease], paraneoplastic, demyelinating, and inflammatory), and idiopathic. Classifications of the ataxias have evolved over time. Originally based on neuropathologic criteria, more recently, a clinical classification was introduced (Harding AE. Lancet 1983;21:1151-1155). The latest classification is genotypebased and presents a challenge in distinguishing the various clinical and hereditary characteristics of multiple subtypes and choice of genetic test. With rare ataxia variants, a complete ataxia battery at a cost of $-\$ 8000$ sometimes seems more appropriate than the selection of one test at $\$ 600$ or a smaller battery, among a total of 17 tests offered, with selection based on the clinical syndrome. Having ruled out symptomatic causes that may be treatable, the diagnosis of hereditary ataxia is important for prognosis, genetic counseling, and possible therapeutic interventions. A diagnostic algorithm presented for adult onset cerebellar ataxias (Brusse E et al. Clin Genet 2007;71:12-24) may overlap with childhood ataxias, but during childhood, congenital and metabolic disorders are more frequent. In some cases, the progression of clinical ataxia and MRI evidence of cerebellar atrophy may be very slow and imperceptible. The sudden onset of strabismus or other oculomotor sign is a more definite marker of progression, requiring immediate specific genetic and prognostic classification preliminary to corrective surgery.

In the above case of pontocerebellar hypoplasia with spinal muscular atrophy the slow progression of ataxia is unusual, since most reports indicate a rapid progression with death in infancy (Barth PG. Brain Dev 1993;1993;15:411-422). A Pubmed search of the literature uncovered several cases of olivopontocerebellar atrophy associated with carbohydrate-deficient glycoprotein (CDG) syndrome type 1 that was recognized at a later age (Pavone L et al. Jrnl Neurol 1996;243:700-705). The serum transferrin test for CDG syndrome would be of interest in the above case (Assmann B et al. Neuropediatrics 2001;32:313-318). 